\title{
Andrew Holden, Jehovah's Witnesses. Portrait of a Contemporary Religious Movement
}

Londres-New York, Routledge, 2002. 206 p. (bibliogr., glossaire, index)

Régis Dericquebourg

\section{OpenEdition}

\section{Journals}

Édition électronique

URL : http://journals.openedition.org/assr/961

DOI : $10.4000 /$ assr.961

ISSN : $1777-5825$

Éditeur

Éditions de l'EHESS

Édition imprimée

Date de publication : 1 octobre 2003

Pagination : 63-170

ISBN : 2-222-96739-2

ISSN : 0335-5985

\section{Référence électronique}

Régis Dericquebourg, «Andrew Holden, Jehovah's Witnesses. Portrait of a Contemporary Religious Movement », Archives de sciences sociales des religions [En ligne], 124 | octobre - décembre 2003, document 124.63, mis en ligne le 25 octobre 2005, consulté le 24 septembre 2020. URL : http:// journals.openedition.org/assr/961 ; DOI : https://doi.org/10.4000/assr.961 
psychothérapeutiques de la religion ou de la spiritualité comme objet d'étude : un chapitre est consacré à la «spiritualité psychologique» et à la naissance de la "pastoral psychology».

Le deuxième intérêt provient du but dans lequel l'ouvrage a été rédigé : comme une introduction destinée à un large public (ce que l'A. précise en appendice): les chapitres thématico-historiques (religion méthaphysique; médecines alternatives; relations entre psychologie et spiritualité) et un index bien fourni (plus de 550 entrées) devraient permettre de remplir ce but.

\section{Baptiste Coulmont.}

Les Origines des sœurs de Saint-Joseph au $\mathrm{XVII}^{\mathrm{e}}$ siècle. Histoire oubliée d'une fondation, Saint-Flour-Le Puy (1641-1650-1661). Paris, Cerf, 2000, 608 p. (bibliogr., index, illustr.) (coll. « Petits-Cerf-Histoire »).

M.-L.G. est bien connue par la maîtrise avec laquelle elle a mené ses recherches théologiques et historiques sur Jeanne-Marie Guyon. Elle nous livre aujourd'hui un instrument de travail très élaboré sur une autre facette de la spiritualité française à l'époque classique. Son investigation de première main dans les archives des origines d'une congrégation féminine dépasse l'intérêt d'une monographie approfondie. En même temps, ayant adopté comme démarche d'exposition celle même de la découverte, l'auteure nous fournit une très utile leçon de méthode.

La fondation des sœurs de Saint-Joseph au milieu du XVII ${ }^{\mathrm{e}}$ siècle a engendré une postérité considérable; encore aujourd'hui, la quarantaine de congrégations qui en sont issues comprend 20000 religieuses dans 56 pays. Un examen extrêmement minutieux des conditions dans lesquelles une telle initiative émerge durant la première moitié du XVIIe éclaire la dynamique religieuse de la période ; cette innovation institutionnelle marquée par l'œuvre de François de Sales combine l'héritage de la mystique rhéno-flamande avec l'influence décisive d'un jésuite. La ligne de ce dernier, le père Médaille, ne se comprendrait pas sans la conjoncture de la «nouvelle spiritualité jésuite » mise à jour pour cette période par Michel de Certeau.

Comme on le voit, le travail présenté par M.-L.G. est particulièrement stimulant quand on y trouve à la fois une différenciation très fine des courants spirituels et un repérage précis de la fécondation croisée qui engendre leurs postérités dans des parturitions qui sont loin d'être sans douleurs. À un moment où l'expérience mystique dut se faire très discrète, «l'autre est la médiation nécessaire qui rend possible l'action et la parole. La relation apostolique seule [...] permet d'articuler l'expérience mystique » (Michel de Certeau). M.-L.G. ajoute: "Pour le père Médaille, ce sont les femmes, les illettrés, le pauvre peuple des campagnes qui l'appellent hors du cercle où la mystique a été asséchée » (p. 507). Ce brassage des divergences aboutit à une certaine harmonie, que l'A. nous donne à voir en analysant deux tableaux peints par Georges de la Tour : «Le soleil des Rhénans chasse le noir de la désespérance humaine, le monde angélique se fait féminin et enfantin et fréquente la «Trinité créée », et le zèle ignatien se marie à la douceur salésienne dans la gamme des tons or et rouge brun » (p. 527).

Jacques Maître.

124.63

HOLDEN (Andrew).

Jehovah's Witnesses. Portrait of a Contemporary Religious Movement. Londres-New York, Routledge, 2002. 206 p. (bibliogr., glossaire, index).

Dans son introduction, l'auteur annonce une étude ethnographique des Témoins de Jéhovah fondée sur une observation participante avouée et sur des entretiens, destinée à appréhender leur vision de la réalité. Toutefois, nous ne décelons pas vraiment une description fine des pratiques et des conduites des fidèles. En fait, l'ouvrage s'apparente plus à une étude sociologique puisque A.H. examine le jéhovisme à la lumière des théories de la modernité et qu'il reprend des questions qui parcourent les études sur les groupes religieux minoritaires comme celles des rapports entre les fidèles et la société ambiante, les causes de la conversion, les conditions sociales et économiques qui ont entouré la naissance de ce mouvement, les réactions à l'échec des prophéties, le contrôle social interne et la régulation des croyances. Finalement l'ouvrage se situe dans la lignée de la thèse de James Beckord, Trumpet of Prophecy: A sociological study of the Jehovah's witnesses (Oxford, Basic, Blackwell, 1975) sans présenter l'avantage d'être la première étude sociologique publiée sur ce mouvement. Nous ne le critiquerons pas sur ce point car il est bon que des études de sciences sociales rappelant les éléments essentiels du jéhovisme paraissent de temps à autre. En revanche, nous le critiquons sur plusieurs points. 
En premier lieu, il paraît maintenant dépassé de commencer un écrit sur le jéhovisme en affirmant que les Témoins de Jéhovah sont négligés par les universitaires. L'A. ne connaît pas les écrits italiens, français, belges et allemands qui ont déjà posé les problèmes qu'il évoque. Il ne se réfère qu'aux écrits anglais et américains. Le Néerlandais Singelenberg est cité car il publie en anglais. En second lieu, A.H. ne traite pas - ou peu - de l'évolution des Témoins de Jéhovah telle que les nouvelles implications dans l'espace public (l'action humanitaire, les engagements dans les associations), la remise en cause d'un contrôle sur la sexualité des couples, les relations moins tendues, voire le dialogue, avec les instances politiques nationales et locales dans les pays occidentaux. Ainsi la question d'une installation durable dans le monde - et le passage éventuel de la secte à la dénomination - après l'échec des annonces eschatologiques ne sont pas posés. En troisième lieu, l'A. semble considérer le jéhovisme comme un fondamentalisme (pp. 40-41). Or, les Témoins sont des biblicistes mais pas des fondamentalistes et encore moins des intégristes. Ils prennent certains versets bibliques à la lettre et en prennent d'autres d'une manière métaphorique. Comme l'a montré Jean Séguy dans un article ancien («De l'usage de la Sainte Bible chez les Témoins de Jéhovah », Concilium, décembre 1967, pp. 147-149), leur lecture des Écritures est transversale. Pour examiner un sujet, on saute d'un verset à un autre en fonction d'une trame préétablie. Quatrièmement, la notion de Témoins comme peuple à part doit être référée au type secte. Enfin, l'émotion et la chaleur affective dans le groupe devraient être relativisées. La rationalité de l'organisation passe avant l'émotion de groupe. Le point fort de l'ouvrage est la mise en évidence d'une attitude paradoxale envers la modernité. A.H. montre qu'ils en refusent certains aspects tandis qu'ils en adoptent d'autres tels que la rationalité de la vie religieuse et le recours systématique aux nouvelles technologies (depuis l'origine).

En dépit de ces remarques, nous considérons ce livre comme une bonne approche du jéhovisme. La présence d'un glossaire est appréciable.

\section{Régis Dericquebourg.}

HUBERT (Ollivier).

Sur la terre comme au ciel. La gestion des rites par l'Église catholique du Québec (fin XVII ${ }^{\mathrm{e}}$-mi XIX ${ }^{\mathrm{e}}$ siècle). Québec, Presses de l'Université Laval, 2000, 232 p. (coll. « Religions, cultures et sociétés »).

Outre leur intérêt spécifique, les travaux sur le Québec ont aussi pour le lecteur français le mérite supplémentaire d'offrir tout à la fois une forme de décentrement et de miroir dans un jeu de balance entre le proche et le lointain. Dans la lignée des travaux de S. Gagnon, R. Brodeur, B. Caulier, entre autres, cette page de l'histoire religieuse et culturelle de la « Belle Province», bien avant la Révolution tranquille, en témoigne. D'emblée cette thèse d'histoire entend dialoguer avec la démarche des anthropologues et se garde de considérer le rite comme seul indice ou instrument de mesure d'une pratique. Refusant également de se laisser enfermer dans une définition figée de la religion populaire opposée à une culture des élites, l'auteur veut saisir la dynamique des rapports de pouvoir dans la gestion et l'institutionnalisation des rites. Dans une analyse fine des sources et des pratiques du XVIII ${ }^{\mathrm{e}}$ et du début du XIX ${ }^{\mathrm{e}}$ siècle, la première partie dresse le cadre par l'étude détaillée du texte du rituel et du discours ecclésiastique. Elle offre ici bien des points de comparaison avec la France de l'époque, mais avec des enjeux parfois différents. Ainsi la concurrence, dans l'ordre de l'efficace, entre rites religieux et rites d'Église prend une coloration particulière dans une région où s'exerce plus vivement la rivalité avec le modèle britannique protestant. Après l'ordre du discours, une seconde partie est consacrée aux structures de contrôle, et porte une forte attention au livre, touchant là l'histoire de l'édition et de l'alphabétisation. On y voit percer progressivement une morale plus inspirée de Liguori, certains vieux rituels sont jugés trop sévères et autoritaires alors que, souligne un sulpicien, en désaccord avec le pouvoir épiscopal, « on n'obéit jamais mieux [à l'autorité] que lorsqu'elle se cache avec le plus grand soin ». Le passage général au rituel romain ne va pas de soi et ne se généralise que dans la seconde moitié du XIX ${ }^{\mathrm{e}}$ siècle. En dernier lieu, l'étude des pôles du contrôle rituel offre des réflexions judicieuses, appuyées sur une lecture nourrie des travaux d'anthropologues, sur le rapport au temps, à l'espace et au corps. L'analyse de la place du Saint Sacrement, en donne un riche exemple, central dans la détermination d'un espace orienté, il «possède à la fois un pouvoir cloisonnant, constructeur d'espace exclusif, et un principe agrégeant, unificateur de lieux, inclusif $»($ p. 258). La gestuelle du rite participe à cette irruption du sacré, et la place du corps est essentielle dans la réalisation du sacrement. Ce corps-temple, sacralisé par le baptême est appelé à partager le Saint Sacrement, mais si l'officiant accomplit 